\title{
Simultaneous Parameter and Input Estimation of a Respiratory Mechanics Model
}

\author{
Francesco Vicario, Antonio Albanese, Dong Wang, \\ Nikolaos Karamolegkos, and Nicolas W. Chbat
}

\begin{abstract}
Real-time noninvasive estimation of respiratory mechanics in spontaneously breathing patients is still an open problem in the field of critical care. Even assuming that the system is a simplistic first-order single-compartment model, the presence of unmeasured patient effort still makes the problem complex since both the parameters and part of the input are unknown. This paper presents an approach to overcome the underdetermined nature of the mathematical problem by infusing physiological knowledge into the estimation process and using it to construct an optimization problem subject to physiological constraints. As it relies only on measurements available on standard ventilators, namely the flow and pressure at the patient's airway opening, the approach is noninvasive. Additionally, breath by breath, it continually provides estimates of the patient respiratory resistance and elastance as well as of the muscle effort waveform without requiring maneuvers that would interfere with the desired ventilation pattern.
\end{abstract}

\section{Introduction}

The need for estimation of the respiratory mechanics is well-known in the medical community $[6,9]$. The mechanical properties of the respiratory system are typically described via two parameters, the resistance $(R)$ and the elastance $(E)$. Quantitative assessment of $R$ and $E$ can aid the clinician to diagnose a respiratory disease, monitor the progression of the disease and the effect of treatment, and optimize

F. Vicario, N. Karamolegkos

Philips Research North America, Briarcliff Manor, NY 10510, USA, and Columbia University, New York, NY 10027, USA,

e-mail: francesco.vicario@philips.com,nikolaos.karamolegkos@philips.com

A. Albanese, D. Wang, N. W. Chbat

Philips Research North America, Briarcliff Manor, NY, 10510, USA,

e-mail: antonio.albanese@philips.com,dong.wang@philips.com, chbat@philips.com 
the ventilator settings to the patient specific needs. The force exerted by the patient to breathe is usually represented as an equivalent pressure generated by the respiratory muscles $\left(P_{m u s}\right)$. The clinical parameter commonly used to assess the effort made by the patient's muscles at each breath is known as work of breathing (WOB). WOB is defined as the mechanical work done by the respiratory muscles during inhalation. It can be computed for every breath as the integral of $P_{m u s}(t)$ over the inhaled volume of air. Knowing the patient WOB is especially important in partially assisted mechanical ventilation modes such as pressure support ventilation (PSV), where patient and ventilator share the mechanical work performed on the respiratory system $[4,12]$. The state of the art for a complete assessment of the respiratory mechanics requires measurement of the esophageal pressure via the insertion of a balloon-tipped catheter in the patient's esophagus [3]. Not only is this technique invasive, but it also needs characterization of the mechanical properties of the chest wall. This generally requires the patient to be made passive (e.g., via sedation or hyperventilation). A well-established technique to assess $R$ and $E$ requires a ventilator maneuver (end inspiratory pause) [8]. Although noninvasive, the maneuver interferes with the normal operation of the ventilator. It is then not suitable for continual monitoring of respiratory mechanics. This is a severe limitation, as in critically ill patients the mechanical properties of the respiratory system can rapidly change. A common noninvasive and maneuver-free method to estimate $R$ and $E$ consists of using the Least Squares (LS) method to fit a suitable mathematical model of the respiratory system to the pressure and flow measurements obtained noninvasively at the patient airway opening $[2,7]$. However, the LS method provides accurate results only if the patient is fully passive. During spontaneous breathing $P_{m u s}$ is no longer a negligible driving force and causes bias in the LS estimates.

The main difficulty in model-based estimation of the respiratory mechanics in spontaneously breathing patients lies with the underdetermined nature of the problem. Besides the model parameters, also part of the input $\left(P_{m u s}\right)$ is unknown. This paper presents an approach to overcome the existence of multiple solutions by constructing an optimization problem subject to physiological constraints to the unknowns to be estimated. Two methods are described, namely constrained optimization (CO) and parametric optimization (PO), and illustrated via an example on animal data.

\section{Description of the Estimation Problem}

\subsection{Mathematical Model of Respiratory Mechanics}

The lungs are traditionally represented as an elastic compartment served by a single resistive pathway. Despite its simplicity, this lumped model is representative of the real lung mechanics and widely accepted in the respiratory research community. The pressure at the entrance of the resistive pathway represents the airway opening 


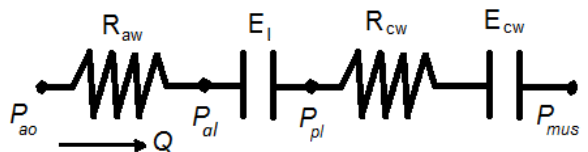

(a)

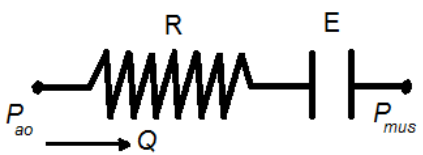

(b)

Fig. 1 Representation of respiratory mechanics by electrical analogues.

pressure $\left(P_{a o}\right)$, whereas the pressure inside the elastic compartment corresponds to the alveolar pressure $\left(P_{a l}\right)$. The alveolar space, in turn, is enclosed in the chest wall that is represented as an additional elastic compartment whose internal pressure corresponds to intrapleural pressure $\left(P_{p l}\right)$. The system is subject to an external pressure $\left(P_{m u s}\right)$ that represents an equivalent pressure of the force exerted by the respiratory muscles. The electrical analogue corresponding to this simplified representation of the respiratory system is shown in Fig. 1a. $R_{a w}$ and $E_{l}$ denote the airways/lungs resistance and elastance, respectively, whereas $E_{c w}$ denotes the elastance of the chest wall. Mechanical dissipation (friction) within the chest wall is taken into account by an additional resistance $R_{c w}$. The simplest model assumes that the resistive and elastic elements in the above electrical analogue are described by constant parameters (linear model). The number of parameters in the electrical analogue in Fig. 1a can be reduced to two, namely the overall resistance $R=R_{a w}+R_{c w}$ and elastance $E=E_{l}+E_{c w}$ of the respiratory system (Fig. 1b) to obtain what is known in the literature as the linear first-order single-compartment model of respiratory mechanics [2]. The air flow $Q(t)$ through the resistive and elastic elements is driven by the pressure difference $P_{a o}(t)-P_{\text {mus }}(t)$. The equation governing its dynamics, known as the equation of motion of the respiratory system, is

$$
P_{a o}(t)=R Q(t)+E V(t)+P_{\text {mus }}(t)+P_{0}
$$

where $V(t)$ represents the volume of air inhaled from the beginning of the breath $(t=0)$, and $P_{0}$ is a constant pressure term balancing the pressure at the airway opening at $t=0\left(V(0)=Q(0)=P_{\text {mus }}(0)=0\right)$.

\subsection{Problem Statement}

The problem addressed in this paper can be stated as follows. Given measurements of pressure and flow at the patient airway opening (i.e., at the mouth or, for patients ventilated with an endotracheal tube, at the so-called Y-juncture), estimate $R, E$, and $P_{m u s}(t)$ in Eq. (1) at each breath. Pressure and flow waveforms are typically available for mechanically ventilated patients, hence the solution to this problem is noninvasive. Also, the waveforms are supposed to come from breaths in normal operative conditions of the ventilator. No additional maneuvers or changes in the ventilation pattern are assumed. 


\subsection{Main Challenges}

As explained in detail in [10], the simultaneous estimation of $R, E$ and $P_{m u s}(t)$ is an underdetermined problem, i.e., there exist infinitely many solutions of triplets $R$, $E, P_{\text {mus }}(t)$ satisfying Eq. (1). Only one of them is the physiological solution that we are after. Indeed, fixing $R$ and $E$ equal to any two values, it is possible to find a pressure waveform $P_{\text {mus }}(t)$ perfectly fitting the data over one breath simply by solving Eq. (1) for $P_{\text {mus }}$. By looking at $P_{a o}-P_{\text {mus }}$ as the input and $V$ or $Q$ as the output of the dynamic system in Fig. 1b, another way to see the underdetermined nature of the estimation problem presented above is that not only are the parameters ( $R$ and $E$ ) to be estimated, but also part of the input $\left(P_{m u s}\right)$. This makes the problem particularly complex.

Another challenge is given by the fact the excitation of the dynamic system in Fig. $1 \mathrm{~b}$ cannot be arbitrarily chosen to facilitate the estimation. The problem has to be solved in operational conditions, with the ventilator supporting a patient according to his/her specific needs. In the above-mentioned PSV mode, the patient initiates the breath by generating a negative $P_{m u s}$. Once the ventilator detects the patient's effort, it triggers and provides pressure at the airway opening trying to match a profile that exponentially approaches the selected PSV pressure level. The ventilator then cycles off (i.e., it stops supporting the patient) typically when the inhaled flow falls below a certain threshold often specified as a fraction of the maximum flow during inhalation. The instant at which the ventilator cycles off is commonly called start of exhalation (SOE).

\section{Approach}

To overcome the underdetermined nature of the mathematical problem, we introduce physiological information on the unknowns to be estimated and exploit it to make the solution to the problem unique. In particular, the signal profile of the pressure exerted by the respiratory muscles does not change arbitrarily over one breath. The patient typically pulls down the diaphragm to inhale, then releases the respiratory muscles to let the diaphragm return to the rest position and no more effort is made until the start of a new breath. This physiological knowledge can be infused in the estimation algorithm in the form of regional constraints on $P_{\text {mus }}(t)$. In this section, we present two methods developed by formulating constraints on $P_{\text {mus }}(t)$ in the three regions of the breath mentioned above: inhalation, muscle relaxation, and rest. 


\subsection{Constrained Optimization (CO)}

$P_{\text {mиs }}(t)$ typically monotonically decreases at the beginning of a spontaneous breath, then monotonically returns to zero as the muscles relax. Finally, in conditions of passive exhalation, this pressure remains zero until the next breath is initiated. These monotonicity regional constraints can be written in the form of inequalities and equalities. For simplicity, the estimation algorithm is formulated below replacing $P_{\text {mus }}(t)$ with $\tilde{P}_{\text {mus }}(t)=P_{\text {mus }}(t)+P_{0}$, since $P_{0}$ is constant over the breath. The estimation problem can then be cast as a constrained optimization problem with cost function

$$
J=\sum_{t_{k}=0}^{t_{k}=(N-1) \Delta t}\left(P_{a o}\left(t_{k}\right)-\left(R Q\left(t_{k}\right)+E V\left(t_{k}\right)+\tilde{P}_{\text {mus }}\left(t_{k}\right)\right)\right)^{2}
$$

to be minimized subject to the following constraints

$$
\begin{array}{ll}
\tilde{P}_{\text {mus }}\left(t_{k+1}\right)-\tilde{P}_{\text {mus }}\left(t_{k}\right) \leq 0 & \text { for } t_{k}=0, \Delta t, \ldots, t_{m}-\Delta t \\
\tilde{P}_{\text {mus }}\left(t_{k+1}\right)-\tilde{P}_{\text {mus }}\left(t_{k}\right) \geq 0 & \text { for } t_{k}=t_{m}, t_{m}+\Delta t, \ldots, t_{q}-\Delta t \\
\tilde{P}_{\text {mus }}\left(t_{k+1}\right)-\tilde{P}_{\text {mus }}\left(t_{k}\right)=0 & \text { for } t_{k}=t_{q}, t_{q}+\Delta t, \ldots, t_{N-1}
\end{array}
$$

where $t_{k}=k \Delta t$ denotes the $(\mathrm{k}+1)^{\mathrm{th}}$ time sample, since the data are typically collected via sampling devices, and $N$ is the total number of time samples in the breath $(k=0,1, \ldots, N-1)$. The parameters $t_{m}$ and $t_{q}$ define the borders of the three regions with different monotonicity (Fig. 2a). The cost function is of LS type, since the squared terms correspond to the difference between the measured $P_{a o}$ and the one estimated from the model in Eq. (1) at each time sample. The unknowns over which $J$ is minimized are $R, E, \tilde{P}_{m u s}\left(t_{0}\right), \tilde{P}_{m u s}\left(t_{1}\right), \ldots, \tilde{P}_{m u s}\left(t_{N-1}\right)$. The constrained optimization problem in Eqs. (2)-(3) is characterized by a quadratic cost function and linear constraints. It belongs to the class of so-called quadratic programs. Wellestablished algorithms such as the interior-point and active-set methods exist to solve this class of optimization problems and routines are available in most commercial software, e.g., Matlab. In the quadratic program in Eqs. (2)-(3), the time parameters $t_{m}$ and $t_{q}$ need be specified. A search for the optimal $t_{m}$ and $t_{q}$ is then necessary. Because in normal conditions the ventilator cycles off when or after the patient effort terminates, we fix $t_{q}=S O E$ and perform a search for $t_{m}$ over the interval $0<t_{m}<S O E$. For each candidate value for $t_{m}$, we solve a quadratic program in the form of Eqs. (2)-(3) and obtain a corresponding minimized value $J^{(m)}$ of the cost function in Eq. (2). The solution arising from the value of $t_{m}$ giving the minimum among all the minimized $J^{(m)}$ 's provides the desired estimates of $R, E$, and $\tilde{P}_{m u s}(t)$. Finally, the estimate of $P_{\text {mus }}(t)$ is extracted from $\tilde{P}_{\text {mиs }}(t)$ by shifting the latter so that the estimate of $P_{\text {mus }}(t)$ is zero in the rest region of the breath (the shift corresponds to $P_{0}$ in Eq. (1)). 


\subsection{Parametric Optimization (PO)}

In the literature on respiratory mechanics, several attempts have been made to describe $P_{\text {mus }}(t)$ over a breath via an explicit mathematical function of time, for instance for the purpose of simulation [1] or emulation [5]. This is a significant simplification of the complex neuronal and physiological phenomena behind the generation of the muscle force responsible for spontaneous breathing. Nevertheless, from an estimation perspective, such attempts inspired simple yet realistic mathematical templates that can be used to represent $P_{\text {mus }}(t)$ with a small number of parameters. Parametrization of $P_{\text {ти }}(t)$ provides another way to overcome the underdetermined nature of the estimation problem addressed in this paper.

As an example, let us assume that $P_{\text {mus }}(t)$ changes linearly with time during inhalation as well as during muscle relaxation (with a different slope) and then remains constant until the next breath initiates. Also assume that the switching time between the three regions, namely $t_{m}$ and $t_{q}$, are known. We can then write $\tilde{P}_{\text {mus }}(t)=P_{\text {mиs }}(t)+P_{0}$ as the following piecewise linear function

$$
\tilde{P}_{m u s}(t)= \begin{cases}P_{q}\left(1-\frac{t}{t_{m}}\right)+P_{m} \frac{t}{t_{m}} & 0 \leq t<t_{m} \\ P_{q}\left(1+\frac{t-t_{q}}{t_{q}-t_{m}}\right)+P_{m}\left(1-\frac{t-t_{m}}{t_{q}-t_{m}}\right) & t_{m} \leq t<t_{q} \\ P_{q} & t_{q} \leq t \leq t_{N-1}\end{cases}
$$

For the sake of clarity, $P_{q}$ corresponds to $P_{0}$ in Eq. (1). Equation (4) provides a family of linear piecewise templates for $\tilde{P}_{m u s}(t)$. Every different pair of $t_{m}$ and $t_{q}$ such that $t_{m}<t_{q} \leq S O E$ provides a template parameterized via the parameters $P_{m}$ and $P_{q}$ (Fig. 2b). For a given pair $\left(t_{m}, t_{q}\right)^{(i)}$, the estimation of $R, E, \tilde{P}_{\text {mиs }}\left(t_{0}\right), P_{\text {mиs }}\left(t_{1}\right), \ldots, P_{\text {mиs }}\left(t_{N-1}\right)$ over the breath reduces to the estimation of $R, E, P_{m}, P_{q}$. The following set of equations can be written for the entire breath

$$
\left[\begin{array}{c}
P_{a o}(0) \\
\vdots \\
P_{a o}\left(t_{m}-\Delta t\right) \\
P_{a o}\left(t_{m}\right) \\
\vdots \\
P_{a o}\left(t_{q}-\Delta t\right) \\
P_{a o}\left(t_{q}\right) \\
\vdots \\
P_{a o}\left(t_{N-1}\right)
\end{array}\right]=\left[\begin{array}{cccc}
Q(0) & V(0) & 1-\frac{0}{t_{m}} & \frac{0}{t_{m}} \\
\vdots & \vdots & \vdots & \vdots \\
Q\left(t_{m}-\Delta t\right) & V\left(t_{-} \Delta t\right) & 1-\frac{t_{m}-\Delta t}{t_{m}} & \frac{t_{m}-\Delta t}{t_{m}} \\
Q\left(t_{m}\right) & V\left(t_{m}\right) & 1+\frac{t_{m}-t_{q}}{t_{q}-t_{m}} & 1-\frac{t_{m}-t_{m}}{t_{q}-t_{m}} \\
\vdots & \vdots & \vdots & \vdots \\
Q\left(t_{q}-\Delta t\right) & V\left(t_{q}-\Delta t\right) & 1+\frac{t_{q}-\Delta t-t_{q}}{t_{q}-t_{m}} & 1-\frac{t_{q}-\Delta t-t_{m}}{t_{q}-t_{m}} \\
Q\left(t_{q}\right) & V\left(t_{q}\right) & 1 & 0 \\
\vdots & \vdots & \vdots & \vdots \\
Q\left(t_{N-1}\right) & V\left(t_{N-1}\right) & 1 & 0
\end{array}\right]\left[\begin{array}{c}
R \\
E \\
P_{q} \\
P_{m}
\end{array}\right]
$$

and solved by the ordinary LS method. The so obtained LS estimates, denoted as $R^{(i)}, E^{(i)}, P_{m}^{(i)}, P_{q}^{(i)}$, are associated with the sum of the least squares $J^{(i)}$. Further templates for $P_{m u s}(t)$ can be constructed by assuming during inhalation and muscle relaxation profiles other than linear. In the demonstration of the PO method given 

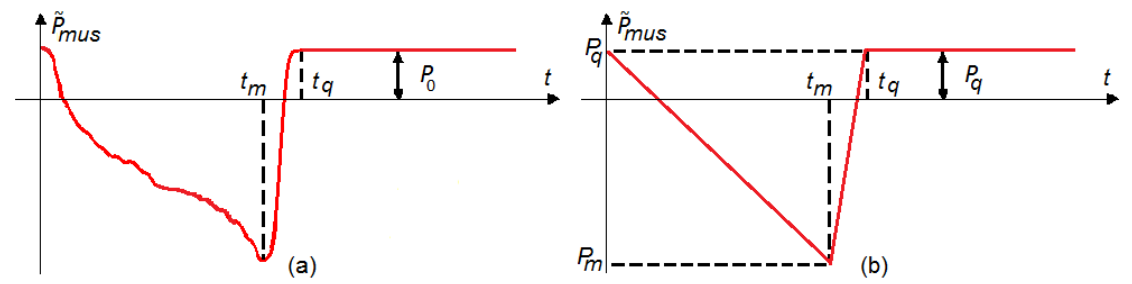

Fig. 2 Regional constraints on respiratory muscle pressure: monotonicity constraints in $\mathrm{CO}$ (a) and linear piecewise cojnstraints in PO (b).

in Section 5, additional parabolic piecewise templates are used for $P_{m u s}(t)$. The presented PO method involves the solution of many ordinary LS problems similar to the one in Eq. (5) for each template ${ }^{(i)}$. Finally, and similar to the $\mathrm{CO}$ method, the estimates of $R, E$, and $P_{m u s}(t)$ for the breath are chosen as those obtained via the $P_{\text {mus }}$ template corresponding to $J_{\min }=\min \left(J^{(1)}, J^{(2)}, \ldots, J^{(L)}\right)$, where $L$ is the total number of templates.

\section{Experimental Results}

To verify the effectiveness of the presented estimation approach with real data, the $\mathrm{PO}$ and $\mathrm{CO}$ methods have been retrospectively tested on available experimental data. The data were collected as part of an educational study performed at the Pulmonary Research and Animal Laboratory at Duke University Medical Center on an adult male pig. The experimental protocol was approved by the local institutional review board committee. During the study, a pig was anesthetized, intubated and connected to an Esprit ventilator with NM3 respiratory monitor (Philips-Respironics). Airway pressure $\left(P_{a o}\right)$ and flow $(Q)$ were measured at the Y-juncture between the breathing circuit and the endotracheal tube, via the standard proximal sensors of the NM3 monitor. An esophageal balloon was used to measure the pressure inside the esophagus $\left(P_{e S}\right)$ as a surrogate of intrapleural pressure. Data were acquired and collected at $100 \mathrm{~Hz}$. The pig was subject to continuous positive airways pressure (CPAP) with variable levels of pressure support ventilation (PSV mode). The measured $P_{a o}$ and $Q$ data are shown in Fig. 3 (top graphs). The performance of the presented algorithms was evaluated by comparing the estimates of $R, E$, and $P_{m u s}(t)$ that were noninvasively estimated via the $\mathrm{CO}$ and $\mathrm{PO}$ methods with those that were invasively calculated from the measured $P_{e s}$ over the same dataset. The latter were considered the gold standard values of $R, E$, and $P_{m u s}(t)$. Such invasive estimates were obtained breath by breath by applying the ordinary LS method to the portion of the system between $P_{a o}$ and $P_{p l}$ in Fig. 1a. This yielded the gold standard estimates $R_{a w}^{G S}$ and $E_{l}^{G S}$ of $R_{a w}$ and $E_{l}$. The gold standard estimates of $R_{c w}$ and $E_{c w}$ were obtained at the end of the educational study by hyperventilating the pig to make it passive $\left(P_{m u s}=0\right)$. This made it possible to find $R_{c w}^{G S}$ and $E_{c w}^{G S}$ by applying the ordinary LS method 

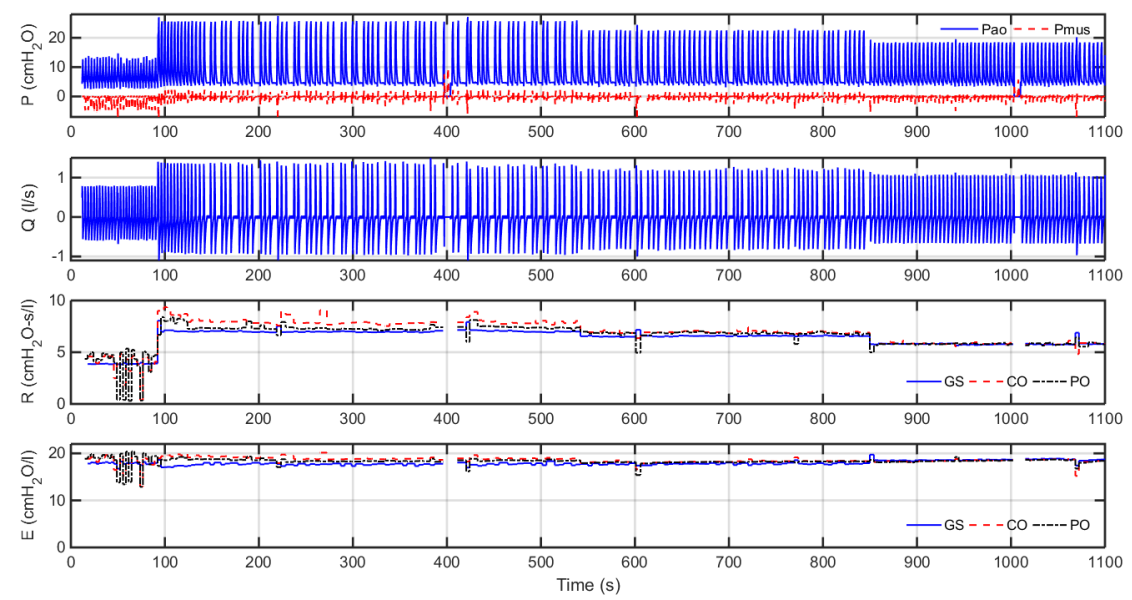

Fig. 3 Experimental dataset and comparison of the estimates of $R$ and $E$ from $\mathrm{CO}$ and PO methods vs. the gold standard (GS).
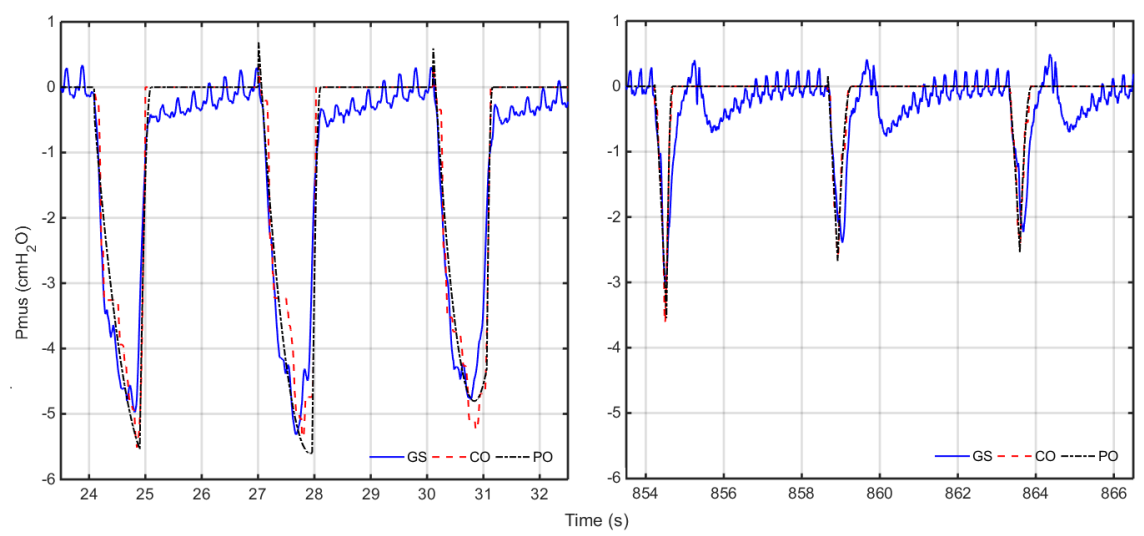

Fig. 4 Comparison of the estimates of $P_{m u s}$ from CO and PO methods vs. the gold standard (GS).

to the portion of the system between $P_{p l}$ and $P_{m u s}$ (with $P_{m u s}=0$ ) in Fig. 1a. The values of $R_{c w}^{G S}$ and $E_{c w}^{G S}$ were supposed to be constant over the entire dataset. The invasive (gold standard) estimates of $R$ and $E$ were finally obtained breath by breath as $R^{G S}=R_{a w}^{G S}+R_{c w}^{G S}$ and $E^{G S}=E_{l}^{G S}+E_{c w}^{G S}$.

The bottom graphs in Fig. 3 show the estimates of $R$ and $E$ from the presented $\mathrm{CO}$ and $\mathrm{PO}$ algorithms and the corresponding gold standard. The match is generally good over the entire dataset, except for a few breaths at low pressure support level (between 50 and 80 seconds) where the $\mathrm{CO}$ and $\mathrm{PO}$ estimates are significantly different from the gold standard. Also, although not particularly significant, some bias in the estimates is visible at high pressure support level. These two issues are discussed in the next section. In the top graph of Fig. 3, also the gold standard 
$P_{m u s}$ is plotted. It is obtained from $R_{c w}$ and $E_{c w}$ and the measured $P_{e s}$ signal as $P_{e s}(t)-R_{c w}^{G S} Q(t)-E_{c w}^{G S} V(t)$ (shifted to eliminate the offset in the last part of the breath, where the muscles are expected to be fully relaxed). Since $P_{m u s}$ is a waveform and not a single-valued parameter over a breath like $R$ and $E$, the comparison between the invasive gold standard and the noninvasive $\mathrm{CO}$ and PO estimates of $P_{m u s}$ cannot effectively be shown over the entire dataset in a single figure. As an example, we show the comparison over two representative segments of the dataset, where the amplitude of $P_{m u s}$ is significantly different (Fig. 4). Both the $\mathrm{CO}$ and PO estimates are able to catch the difference in the depth of the respiratory muscle effort. The interested reader is also referred to [11] where the estimated $P_{\text {mus }}$ signal is demonstrated to be valuable in computing the WOB breath by breath.

\section{Discussion}

The paper presented two methods for the simultaneous estimation of respiratory resistance, elastance and muscle effort in spontaneously breathing patients under mechanical ventilation. The two methods follow the same approach based on a physiological model and physiological constraints on the respiratory muscle pressure signal to be estimated over the breath. As such, they provide the clinicians with valuable insight into respiratory mechanics. Compared to existing techniques, the new approach is noninvasive and does not require maneuvers interfering with the desired ventilation patterns. Continual noninvasive assessment of respiratory mechanics holds the promise that clinicians will be able to better provide mechanical ventilator support with fewer adverse effects and ultimately better outcomes.

The results shown in Figs. 3 and 4 reveal that the performance of the $\mathrm{CO}$ and PO methods is very similar. Although the former is more elegant and has more solid theoretical foundation, the latter is computationally less demanding. This is a significant advantage for the envisioned application, where the estimation algorithm needs to run in quasi real time. After collection of pressure and flow data from a breath, the corresponding estimates must be computed before the next breath ends. On average a breath lasts 4 to 5 seconds. On a laptop with $8 \mathrm{~GB}$ of RAM and i7 $\mathrm{CPU}$ at $2.60 \mathrm{GHz}$, the Matlab implementation of the $\mathrm{CO}$ method cannot run in real time, since every breath requires on average 10 seconds to be processed. On the other hand, the PO method takes an average of 2 seconds per breath to run. Despite potential room for optimization in the implementation of the presented estimation methods, $\mathrm{PO}$ has a significant advantage in (quasi) real-time applications.

Being based on the same approach, $\mathrm{PO}$ and $\mathrm{CO}$ share similar limitations that are visible in the dataset in Fig. 3. Between 50 and 80 seconds, there are a few breaths where both estimates are significantly far from the gold standard. As proven in [10] for the $\mathrm{CO}$ method, the estimation error arises from the constraints not being able to make the solution to the estimation problem unique when the ventilator cycles off before the respiratory muscles have completely relaxed. PO shows a behavior similar to CO. This condition is more likely to occur at low PSV levels, where the venti- 
lator does not sufficiently support the patient. Additionally, again with reference to Fig.3, one can notice that when the gold standard $P_{m u s}$ signal presents some positive spikes the estimates of $R$ and $E$ tend to be biased. Indeed, between 100 and $540 \mathrm{sec}-$ onds (where the pressure support level is highest) and to a lesser extent between 540 and 850 seconds (slightly lower ventilator support), the gold standard $P_{m u s}$ becomes significantly positive during exhalation. This indicates a condition of active patient exhalation that violates the assumptions behind the regional constraints formulated for both the $\mathrm{CO}$ and $\mathrm{PO}$ methods. As a consequence, bias affects the estimates and its magnitude depends on how intense the active exhalation is. As discussed in [11], both early cycling off and active exhalation are forms of asynchrony between the patient and the ventilator. As such, they are undesired from a clinical point of view and they should be eliminated regardless of respiratory mechanics estimation. An appropriate choice of ventilator settings made by the clinician would then be beneficial not only to the patient but also to the $\mathrm{CO}$ and $\mathrm{PO}$ methods.

\section{References}

1. Albanese, A., Cheng, L., Ursino, M., Chbat, N.W.: A comprehensive mathematical model of the human cardiopulmonary system: model development. Am. J. Physiol. (submitted for publication)

2. Bates, J.H.: Lung Mechanics: An Inverse Modeling Approach (Cambridge University Press, Cambridge, UK, 2009), pp. 37-61

3. Benditt, J.O.: Esophageal and gastric pressure measurements. Resp.Care, 50(1), 68-77 (2005)

4. Brochard, L., Harf, A., Lorino, H., Lemaire, F.: Inspiratory pressure support prevents diaphragmatic fatigue during weaning from mechanical ventilation. Am. Rev. Respir. Dis., 139, 513-521 (1989)

5. Fresnel, E., Muir, J.-F., Letellier, C.: Realistic human muscle pressure for driving a mechanical lung. EPJ Nonlinear Biomed. Phys., 2(7), (2014) doi:10.1140/epjnbp/s40366-014-0007-8

6. Grinnan, D.C., Truwit, J.D.: Clinical review: respiratory mechanics in spontaneous and assisted ventilation. Crit. Care., 9(5), 472-484 (2005)

7. Iotti, G.A., Braschi, A., Brunner, J.X., Smits, T., Olivei, M., Palo, A., Veronesi, R.: Respiratory mechanics by least squares fitting in mechanically ventilated patients: application during paralysis and during pressure support ventilation. Intensive Care Med., 21, 406-413 (1995)

8. Nucci, G., Cobelli, C.: Mathematical models of respiratory mechanics. In: Carson, E., Cobelli, C. (eds.) Modeling Methodology for Physiology and Medicine, $1^{\text {st }}$ ed., ch. X, pp. 279-304. Academic Press, San Diego, CA (2001)

9. Polese, G, Serra, A., Rossi, A.: Respiratory mechanics in the intensive care unit. In: Gosselink, R., Stam, H. (eds.) Eur. Respir. Monogr., 31, 195-206 (2005)

10. Vicario, F., Albanese, A., Karamolegkos, N., Wang, D., Seiver, A., Chbat, N.W.: Noninvasive estimation of respiratory mechanics in spontaneously breathing ventilated patients: a constrained optimization approach. IEEE Trans. Biomed. Eng. (submitted for publication)

11. Vicario, F., Albanese, A., Wang, D., Karamolegkos, N., Chbat, N.W.: Constrained optimization for noninvasive estimation of work of breathing. $37^{\text {th }}$ Conf. Proc. IEEE Eng. Med. Biol. Soc. (EMBC) (to be published)

12. Yamada, Y., Shigeta, M., Suwa, K., Hanaoka, K.: Respiratory muscle pressure analysis in pressure-support ventilation. J. Appl. Physiol., 77(5), 2237-2243 (1994) 